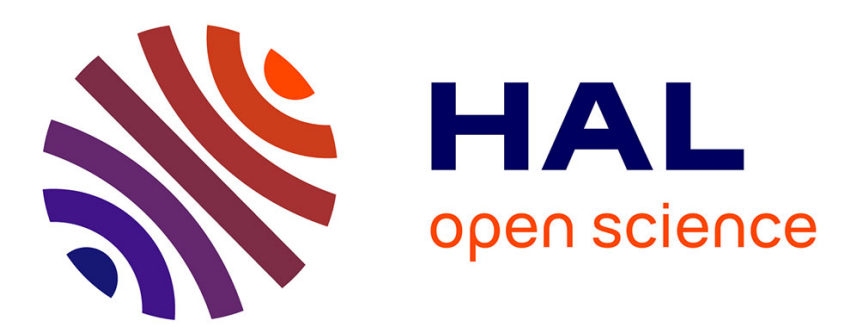

\title{
Integrated three-dimensional scanner for endoscopic optical coherence tomography
}

\author{
K. Aljasem, L. Froehly, H. Zappe, A. Seifert
}

\section{To cite this version:}

K. Aljasem, L. Froehly, H. Zappe, A. Seifert. Integrated three-dimensional scanner for endoscopic optical coherence tomography. Conference on Micro-Optics 2010, Apr 2010, Bruxelles, Belgium. pp.7716, 10.1117/12.851644. hal-00586328

\section{HAL Id: hal-00586328 \\ https://hal.science/hal-00586328}

Submitted on 19 Apr 2021

HAL is a multi-disciplinary open access archive for the deposit and dissemination of scientific research documents, whether they are published or not. The documents may come from teaching and research institutions in France or abroad, or from public or private research centers.
L'archive ouverte pluridisciplinaire HAL, est destinée au dépôt et à la diffusion de documents scientifiques de niveau recherche, publiés ou non, émanant des établissements d'enseignement et de recherche français ou étrangers, des laboratoires publics ou privés. 


\title{
Integrated three-dimensional scanner for endoscopic optical coherence tomography
}

\author{
Khaled Aljasem ${ }^{1}$, Luc Froehly ${ }^{2}$, Hans Zappe ${ }^{1}$, and Andreas Seifert ${ }^{1}$ \\ ${ }^{1}$ Department of Microsystems Engineering - IMTEK, University of Freiburg, GERMANY \\ ${ }^{2}$ Institut FEMTO-ST, UMR CNRS 6174, Université de Franche-Comté, Besançon, FRANCE
}

\begin{abstract}
Numerous optical imaging techniques have been developed for clinical diagnostics; among these, optical coherence tomography (OCT) has proven to be of considerable utility due to its ability to non-destructively image below the surface of tissue. Endoscopic OCT systems will further extend the capabilities of this approach but require an additional means for scanning in two or three dimensions.

We present an integrated optical microsystem which allows scanning of an optical beam in three dimensions (an area scan combined with dynamic focus) suitable for an endoscopic OCT probe. The system is defined by a tunable pneumatically-actuated micro-lens combined with an electrostatically-actuated two-axis micro-mirror, allowing functionality hitherto not achievable.
\end{abstract}

Keywords: Dynamically focusing optical coherence tomography, tunable micro-lens, scanning micro-mirror, endoscopic optical coherence tomography

\section{INTRODUCTION}

Since its first invention in the early 1990s, OCT was significantly improved so that it could be employed for clinical applications. ${ }^{1}$ As macroscopic OCT versions are usable only in external fields of human diagnostics, such as in ophthalmology ${ }^{2,3}$ and dermatology, ${ }^{4,5}$ OCT has to be combined with endoscopic technology for internal human diagnostics.

Endoscopic OCT is based on the integration of a miniaturized lateral-scan system which allows the generation of $2 \mathrm{D}$ or $3 \mathrm{D}$ images. ${ }^{6}$ In addition to the lateral scan, a dynamic focus has been shown to be essential for maintaining a high lateral resolution and high image contrast over the depth scan during imaging. ${ }^{7}$ Dynamic focusing is achieved either by mechanically moving the focusing objectives ${ }^{8}$ or by using tunable micro-optics. ${ }^{9-11}$ Tunable systems have only been demonstrated in macroscopic versions and without a combination with scanning mirrors. In this paper, we present a novel miniaturized three-dimensional (3D) scanner based on a tunable micro-lens combined with a two-dimensional (2D) scanning micro-mirror. The functionality of the 3D scanner and its miniaturized version are demonstrated.

\section{THREE-DIMENSIONAL SCANNING SYSTEM}

The scanning system consists of a tunable micro-lens and a 2D scanning micro-mirror. Whereas the scanning micro-mirror allows a 2D lateral scan, the tunable micro-lens allows an axial scan of the focal point leading to a dynamically adjustable focus.

\subsection{Scanning procedure}

The scanning system manipulates the focal point in three dimensions by combining the lateral and the transverse scan with the dynamic focus. The way of combining these different scans, to achieve a 3D image, depends on the maximum speed achievable in each scanning direction. Figure 1 shows a schematic design of the 3D scanner combining the micro-lens and the micro-mirror. Since the scan-speed of the mirror is much faster than the tunability of the micro-lens, the most convenient scanning procedure results by positioning the focal point to a certain depth before carrying out the lateral scan. After each 2D lateral scan, the focal point is set to a new depth after which another 2D lateral scan is performed.

Further author information: (Send correspondence to Khaled Aljasem)

Khaled Aljasem: E-mail: aljasem@imtek.uni-freiburg.de, Telephone: +49761 2037519 


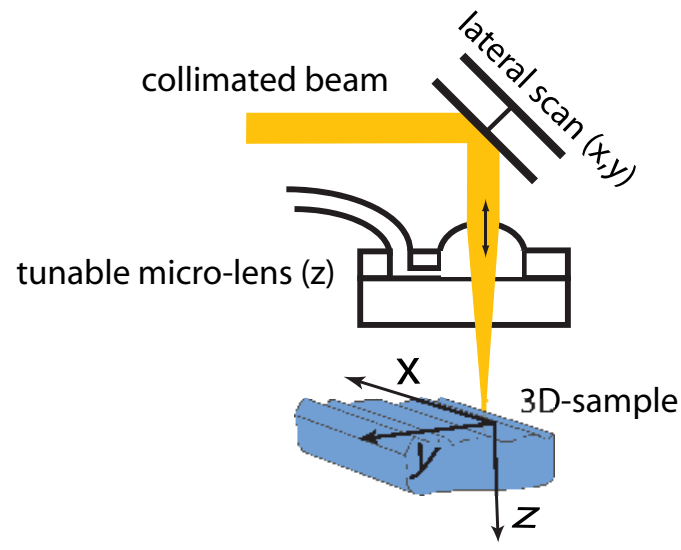

Figure 1. Principle of the 3D scanning system: the beam is first focussed along the z-axis, after which the scanning mirror moves the focused beam laterally in $\mathrm{x}$ - and $\mathrm{y}$-directions.

\subsection{Tunable micro-lens}

The micro-lens consists of a silicon substrate which is structured by photolithography to realize the aperture, liquid-cavities, and a pressure input. A flexible transparent polydimethylsiloxane (PDMS) membrane is attached to the top of the substrate. To seal the lens, the backside of the silicon substrate is bonded onto a transparent glass substrate. Figure 2-a illustrates schematically the functionality of the tunable micro-lens. The lens is filled with a water/ethanol mixture in the ratio 1:1. The PDMS membrane defines the interface between air and the optical liquid. By applying a pneumatic pressure to the bottom channel, as seen in Figure 2-a, the membrane is distended, leading to a convex surface and hence a well-defined focus for the incoming collimated beam which comes from the backside of the lens. By changing the pressure, the curvature of the membrane varies, and the focal length can be tuned.

Figure 2-b shows a photo of the assembled micro-lens. The aperture of the lens may be varied according to the design requirements. The diameter of the aperture determines the diameter of the collimated beam transmitted through the lens and must thus be larger than the collimated beam. The advantage of pneumatic actuation for such a tunable lens is that this approach is widely used in clinical endoscopy for diagnostics and therapy ${ }^{12,13}$ and can thus be easily adapted for this additional optical tool.

The lens has a wide focal length tuning range, between $\infty$ to $1 \mathrm{~mm}$. The lateral resolution of this micro-lens is below $15 \mu \mathrm{m}$ for a tuning range of more than $3 \mathrm{~mm}$. The resolution of the entire optical system can be improved to better than $8 \mu \mathrm{m}$ by inserting a high quality commercial lens between the incident collimated beam and the tunable lens. ${ }^{14}$

\subsection{Scanning micro-mirror}

The lateral scan in two directions is realized by a torsional parallel-plate scanning micro-mirror. The mirror uses electrostatic actuation by applying a voltage between the mirror plate and electrodes which are placed below the plate. A lateral scan (e.g. x-axis) is achieved by increasing the voltage along one axis. Additional electrodes are placed below the gimbal to actuate the mirror in the transverse direction (y-axis). Figure 3 shows a photo of an assembled micro-mirror.

The mirror is fabricated by standard bulk-micromachining employing anisotropic silicon etching from the bottom side of a silicon substrate to define an approximately $6 \mu \mathrm{m}$ thick membrane. A selective inductivelycoupled plasma etch (ICP) is applied from the topside of the membrane to define the mirror plate, the torsional beams, and the gimbals. A $200 \mathrm{~nm}$ thick aluminum layer is deposited onto the top of the mirror to improve the reflectivity. The electrodes consist of a aluminum layer which is lithographically defined on a glass substrate onto which the silicon chip is placed. Finally, the integrated scanning mirror is assembled and packaged in a dual-inline-package (DIP) housing with 24 pins. This packaging is very robust and protects the sensitive mirror during extensive laboratory experiments for characterization. 


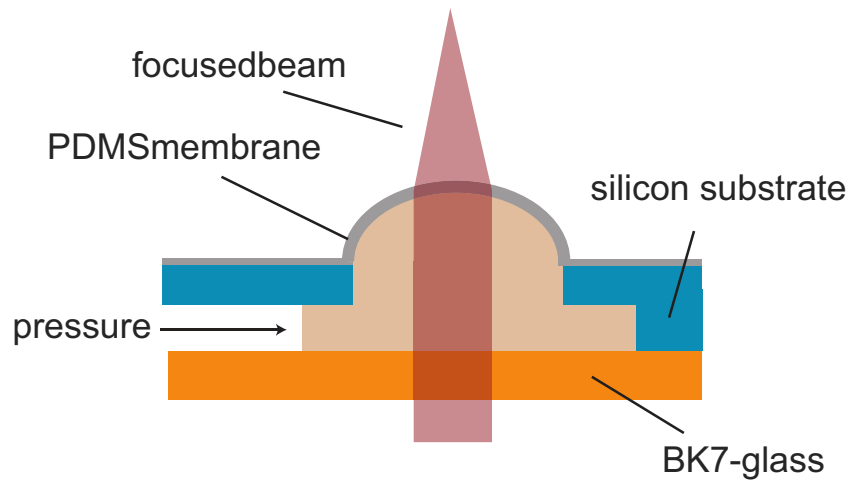

a)

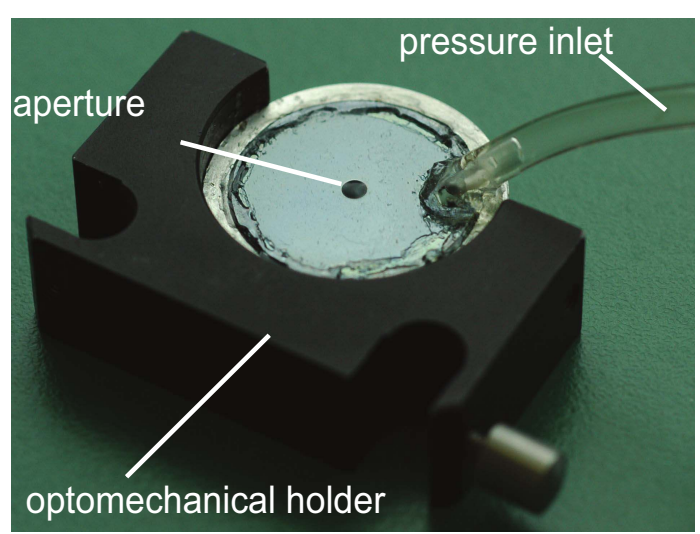

b)

Figure 2. a) Principle of a pneumatically actuated tunable micro-lens. The lens consists of three levels: a PDMS membrane, a silicon substrate, and a BK7 glass plate. The cavity of the lens is filled with a water/ethanol mixture. Actuation is accomplished by applying pressure to the lens cavity. b) Fabricated tunable micro-lens with $2 \mathrm{~mm}$ aperture. The lens is assembled in a mechanical holder to facilitate a proper alignment of the aperture with respect to the optical axis of the OCT system.

a)

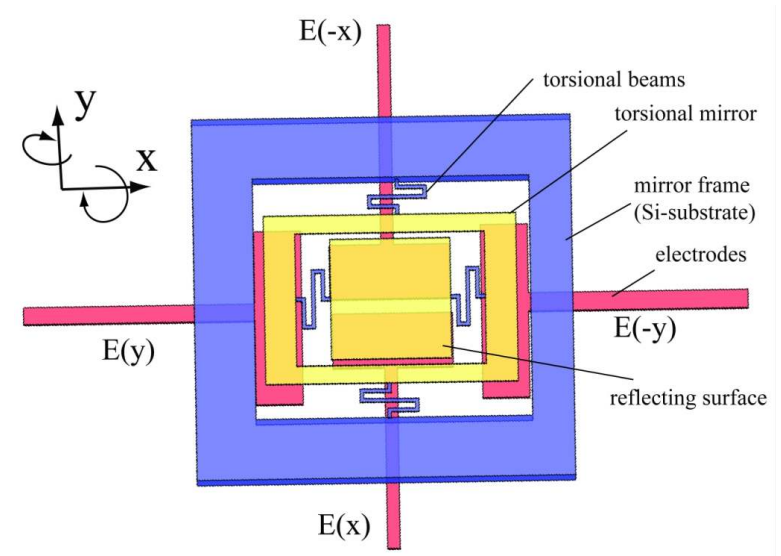

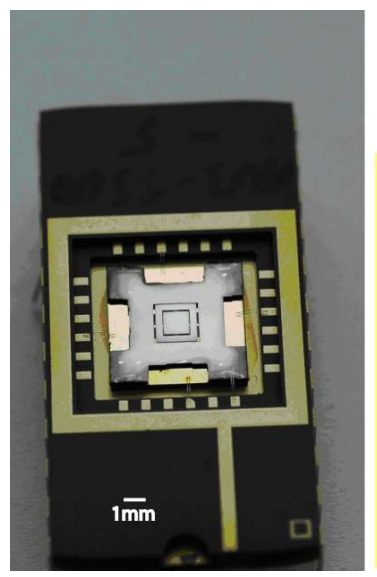

b)

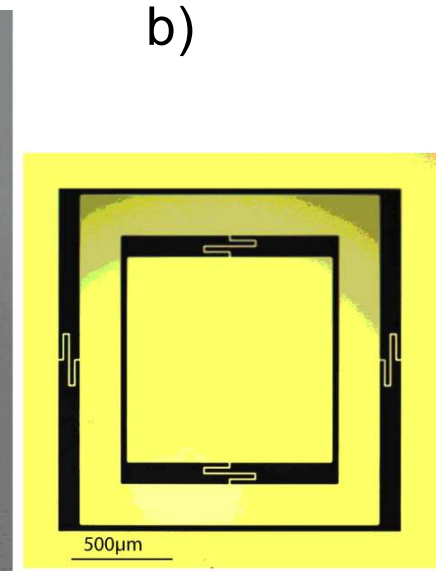

Figure 3. Schematic design of the 2D scanning mirror. a) A 3D gimbal-mounted design in which the mirror is connected by springs to a frame which is in turn suspended from the outer silicon substrate, allowing two-dimensional movement. b) Photo of the mirror packaged for testing purpose and a close-up of the central portion showing the torsional beams.

\subsection{Imaging with three-dimensional scanner}

To test the functionality of the OCT system using this tunable lens and scanning mirror, the 3D scanner was used to illuminate a part of a target by a well-defined spot size. The target consists of a glass substrate with a $200 \mathrm{~nm}$ thick aluminum layer deposited on top. Above the aluminum layer, a $500 \mu \mathrm{m}$ long "F" structure is lithographically defined by selective chemical etching.

The illumination spot is defocussed and the scanning mirror scans this defocused spot over the target vertically and in discrete steps horizontally. The backreflected beam at each point is then imaged onto a CCD camera. Figure 4 shows the image of the entire " $\mathrm{F}$ " after rastering showing that the $2 \mathrm{D}$ scanning system extends the field of vision so that the "F" entirely appears on the detector. 


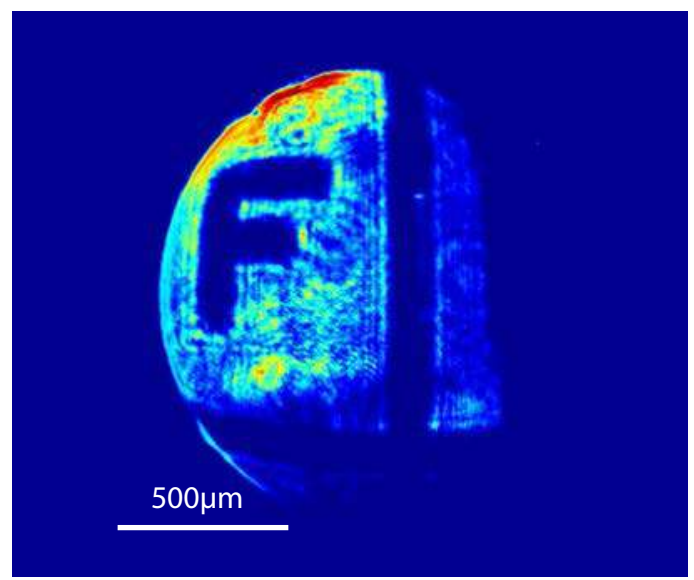

Figure 4. 2D-image of a target generated by manipulation of the focal spot using a dynamic focus and a $2 \mathrm{D}$ lateral scan.

\section{3D SCANNER FOR OPTICAL COHERENCE TOMOGRAPHY}

\subsection{OCT setup and configuration}

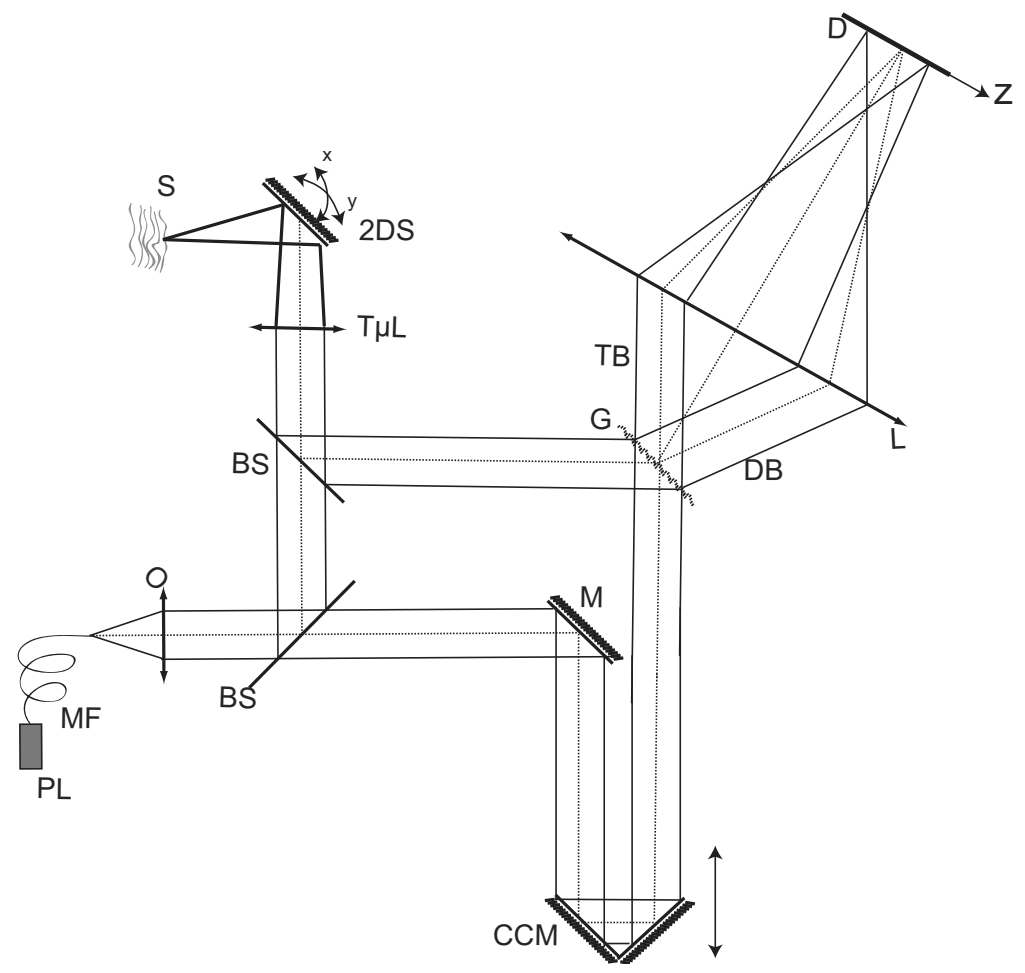

Figure 5. OCT setup for testing micro-optical components: PL Pulsed Laser source, MF Monomode Structured optical fiber, O microscope objective, BS Beam Splitter, M Mirror, CCM Corner Cube Mirror, G Diffraction Grating, L Lens, $T \mu L$ Tunable Micro-Lens, 2DS Two Dimensional micro Scanner, S Sample, TB Transmitted Beam, DB Diffracted Beam, D Detector

The OCT setup used in this experiment was developed at the FEMTO-ST laboratory in Besançon, France. ${ }^{15}$ The setup is depicted in Figure 5 and its working principle is extensively described previously. ${ }^{16}$ Basically, the correlation between the reference arm and the sample arm is directly displayed in the plane of the detector D. In fact, the $\mathrm{z}$ dimension in the $\mathrm{D}$ plane corresponds directly to the depth into the sample (i.e. an A-scan) so that 
the intensity expression in the plane $\mathrm{D}$ is

$$
C(z)=I_{0}+2 \mathcal{R} e\left[\int_{\nu} \hat{R}(\nu) \hat{S}(\nu) e^{-j 2 \pi\left(\frac{2 z}{\gamma c} \sin \theta_{i}\right) \nu} e^{j \frac{4 \pi z}{\gamma \Lambda}} d \nu\right]
$$

where $\mathrm{z}$ is the horizontal coordinate on the CCD camera lines, $I_{0}$ the background intensity, $\mathcal{R} e$ defines the real part, $\hat{R}(\nu)$ is the complex spectrum of the reference field, $\hat{S}(\nu)$ that of the sample field, $\theta_{i}$ is the incidence angle of the diffracted beam onto the grating, $\gamma$ the magnification of the imaging system, and $\Lambda$ the diffraction grating period. Equation 1 clearly shows the correlation operation between the temporal fields realized by the system. The temporal variable is spatially displayed through the variable change.

\subsection{Probe design and realization}

Microsystems engineering allows the miniaturization of the scanning subcomponents so that they can be integrated into endoscopic probes with minimal geometrical dimensions. Figure 6 shows the scanning probe with the integrated scanning micro-mirror and the tunable micro-lens. As can be seen in the figure, the body of the probe is made of aluminum by high precision machining. A circular opening in the body from the left-side allows an insertion of a cylindrical collimator.

The collimated beam with an diameter of $\sim 0.7 \mathrm{~mm}$ propagates through the probe and hits the scanning mirror which is oriented at $45^{\circ}$ with respect to the incident beam. The beam thus passes through the aperture of the tunable micro-lens which focuses it to the desired position on the target. As the aperture is $1.5 \mathrm{~mm}$ in diameter, which is approximately two times larger than the incident collimated beam, a tilting angle of more than $4^{\circ}$ can be realized through the aperture.

To improve the lateral resolution of the probe, a high quality lens with relatively long focal length is inserted in front of the tilting mirror. ${ }^{14}$ By inserting such a lens, the beam is slightly focused before passing through the tunable lens and hence the effect of spherical aberrations of the tunable lens is significantly reduced. The diameter of the focused beam passing the tunable lens becomes smaller by inserting the fixed-focal length lens and accordingly, the maximum titling angle, at which the beam reaches the edge of the lens, increases.

a)

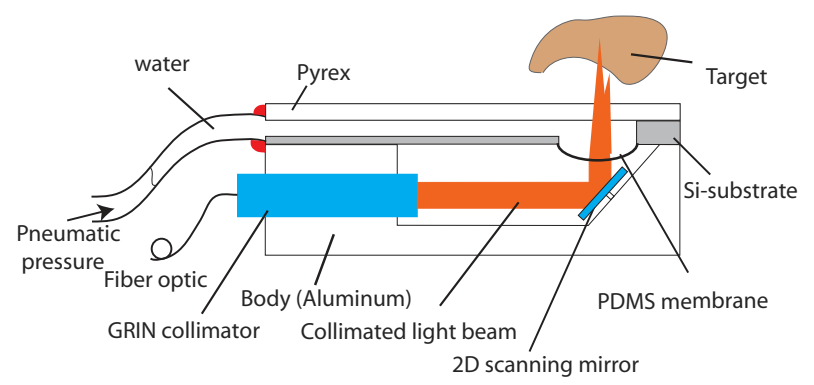

b)

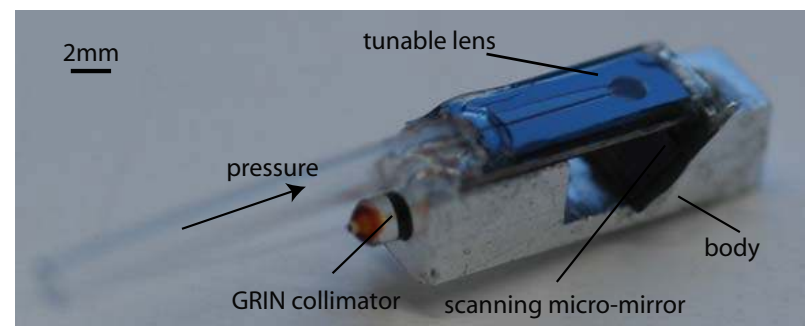

Figure 6. Figure.2: Schematic design (a) and photo (b) of the tunable probe with integrated mirror and micro-lens.

\subsection{Results and discussion}

The OCT setup using this scanning system was employed to obtain images of a biological sample with two different positions of the focal point. A first image is produced by applying a $2 \mathrm{D}$ scan with a focus far from the region of interest, above the sample, yielding a complete 3D OCT image of the sample. By subsequently shifting the focal point deeper into the sample, another $2 \mathrm{D}$ lateral scan is obtained. Figure 7 shows different OCT images obtained by this manipulation of the focal point in three dimensions.

As can be seen in Figure 7-a, the OCT images obtained when sample was far from the focus yields images with low resolution. By tuning the focal point to a reasonable position, slightly below the surface, the image contrast is significantly improved, which leads to a significant improvement of the OCT image quality. The measured lateral resolution of the images was approximately $8 \mu \mathrm{m}$, a value obtained for the entire OCT setup 


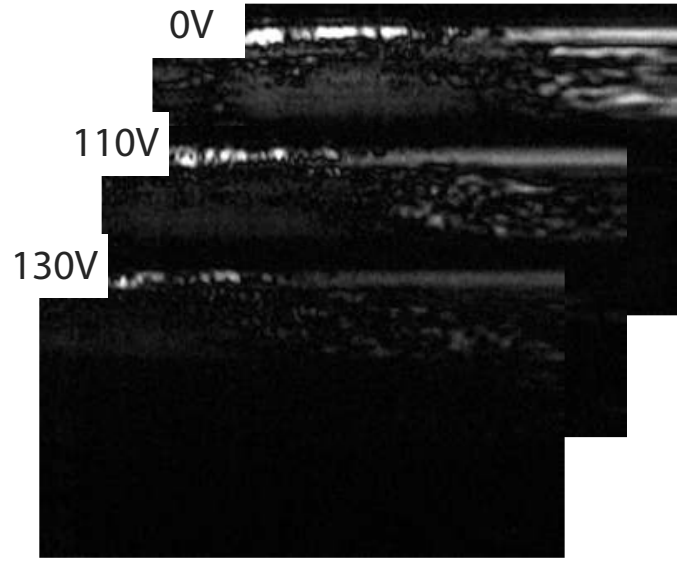

a)

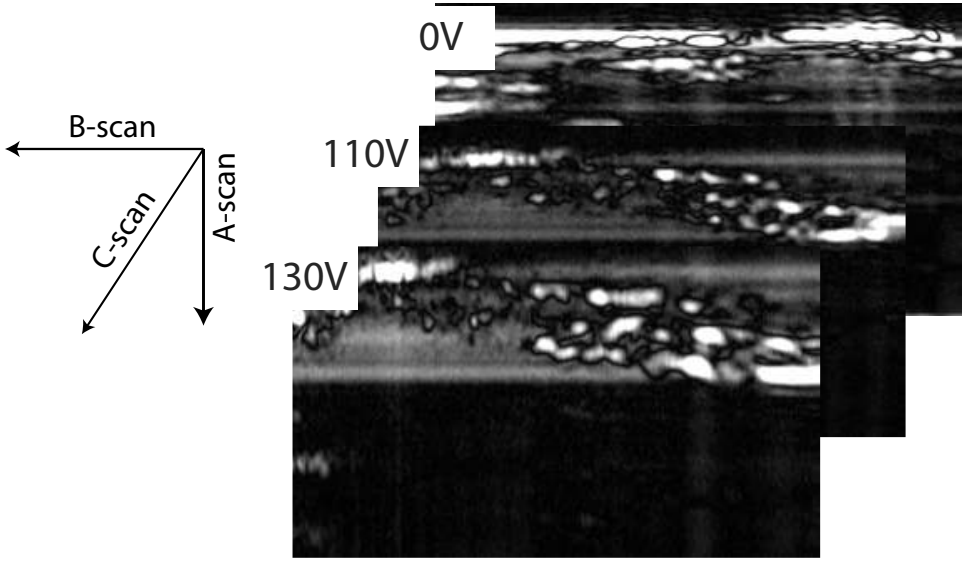

b)

Figure 7. OCT images obtained by manipulating the focal point in three dimensions. The left images represent three B-scans where the sample was out of focus. The right images are obtained while the sample was in focus (the focal point is slightly below the surface of the sample).

with the mirror, the tunable lens, and the image processing. The images shown in Figure 7-b confirm the utility of the tunable micro-lens in improving the image quality without any mechanical adjustment of the target or the objective lens system.

\section{CONCLUSION}

A three-dimensional scanning system for endoscopic OCT applications was presented. The system allows an improvement of 3D OCT images by allowing tuning of the focal point inside the target. The miniaturized scanning probe with a diameter of $4 \mathrm{~mm}$ promises to be an ideal solution for endoscopic imaging.

The miniaturized endoscopic probe opens new opportunities in the field of endoscopy based on micro-optical devices such as micro-lenses and micro-mirrors. Such a probe configuration can be employed not only for OCT, but also for other endoscopic imaging methods or it may be used in conventional monitoring for endoscopy to improve the contrast of endoscopic images as well as to increase the view-angle of endoscopes.

\section{REFERENCES}

[1] A. M. Zysk, F. T. Nguyen, A. L. Oldenburg, D. L. Marks, and S. A. Boppart, "Optical coherence tomography: a review of clinical development from bench to bedside," Journal of Biomedical Optics 12(5), p. 051403, 2007.

[2] T. Schmoll, C. Kolbitsch, and R. A. Leitgeb, "Ultra-high-speed volumetric tomography of human retinal blood flow," Opt. Express 17(5), pp. 4166-4176, 2009.

[3] R. Inoue, M. Hangai, Y. Kotera, H. Nakanishi, S. Mori, S. Morishita, and N. Yoshimura, "Three-dimensional high-speed optical coherence tomography imaging of lamina cribrosa in glaucoma," Ophthalmology 116(2), pp. 214-222, 2009.

[4] X. Liang and S. A. Boppart, "Biomechanical properties of in vivo human skin from dynamic optical coherence elastography," Biomedical Engineering, IEEE Transactions on (Accepted for publication and in a future issue of this journal), 2009.

[5] M. Mogensen, L. Thrane, T. M. Jørgensen, P. E. Andersen, and G. B. E. Jemec, "Oct imaging of skin cancer and other dermatological diseases," Journal of Biophotonics 2(6-7), pp. 442-451, 2009.

[6] Z. Yaqoob, J. Wu, E. McDowell, X. Heng, and C. Yang, "Methods and application areas of endoscopic optical coherence tomography," Journal of Biomedical Optics 11(6), p. 063001, 2006. 
[7] K. Aljasem, A. Werber, A. Seifert, and H. Zappe, "Fiber optic tunable probe for endoscopic optical coherence tomography," Journal of Optics A: Pure and Applied Optics 10(4), p. 044012 (8pp), 2008.

[8] M. Hughes and A. Podoleanu, "Simplified dynamic focus method for time domain OCT," Electronics Letters 45(12), pp. 623-624, 2009.

[9] A. Divetia, T.-H. Hsieh, J. Zhang, Z. Chen, M. Bachman, and G.-P. Li, "Dynamically focused optical coherence tomography for endoscopic applications," Applied Physics Letters 86(10), p. 103902, 2005.

[10] B. Qi, P. A. Himmer, M. L. Gordon, V. X. D. Yang, D. L. Dickensheets, and A. I. Vitkin, "Dynamic focus control in high-speed optical coherence tomography based on a microelectromechanical mirror," Optics Communications 232, pp. 123-128, Mar. 2004.

[11] D. Dickensheets, M. Overcast, P. Himmer, V. Yang, and I. Vitkin, "Focus tracking in time domain optical coherence tomography using membrane mirrors operated near snap-down," Optical MEMS and Their Applications Conference, 2006. IEEE/LEOS International Conference on, pp. 170-171, Aug. 2006.

[12] S. Chuah, T. Hu, K. Wu, P. Hsu, W. Tai, Y. Chiu, C. Lee, and C. Changchien, "Clinical remission in endoscope-guided pneumatic dilation for the treatment of esophageal achalasia: 7-year follow-up results of a prospective investigation," Gastrointestinal Surgery 13(5), pp. 1873-4626, 2009.

[13] H. Itoh, A. Saika, H. Oka, A. Shiotani, T. Hara, S. Nishioka, K. Higashp, and M. Takatsuji, "Endoscopic dilatation therapy using a small caliber balloon for esophageal achalasia," Digestive Endoscopy 10(4), pp. 343-347, 1998.

[14] K. Aljasem, A. Seifert, and H. Zappe, "OCT imaging with high lateral resolution using a dynamically focusing multi-lens system," in Optical Coherence Tomography and Coherence Techniques IV, P. E. Andersen and B. E. Bouma, eds., 7372(1), p. 73721S(6), SPIE, 2009.

[15] L. Froehly, L. Furfaro, P. Sandoz, and P. Jeanningros, "Dispersion compensation properties of grating-based temporal-correlation Optical Coherence Tomography systems," Optics Communications 282, pp. 1488-1495, 2009.

[16] L. Froehly, M. Ouadour, L. Furfaro, P. Sandoz, P. Leproux, G. Huss, and V. Couderc, "Spectroscopic OCT by Grating-Based Temporal Correlation Coupled to Optical Spectral Analysis," International Journal of Biomedical Imaging 2008, pp. 752340-7, 2008. 